\title{
Adolescents who binge eat and drink: The role of emotion regulation
}

\author{
Fiorenzo Laghi, Francesca Liga, and Sara Pompili
}

\section{QUERY SHEET}

This page lists questions we have about your paper. The numbers displayed at left can be found in the text of the paper for reference. In addition, please review your paper as a whole for correctness.

Q1. Please provide the degrees/educational qualifications of all the authors.

Q2. Please check the author names and affiliation details for correctness.

Q3. The ORCID details of the authors have been validated against ORCID registry. please check the ORCID ID details of the authors.

\section{TABLE OF CONTENTS LISTING}

The table of contents for the journal will list your paper exactly as it appears below:

Adolescents who binge eat and drink: The role of emotion regulation

Fiorenzo Laghi, Francesca Liga, and Sara Pompili 


\section{Adolescents who binge eat and drink: The role of emotion regulation}

Q3 Fiorenzo Laghi $^{\mathrm{a}} \mathbb{D}$, Francesca Liga ${ }^{\mathrm{b}}$ (D) , and Sara Pompili ${ }^{\mathrm{a}}$

${ }^{a}$ Department of Developmental and Social Psychology, University of Rome Sapienza, Rome, Italy; ${ }^{b}$ Department of Clinical and Experimental Medicine, University of Messina, Messina, Italy
Q1

\section{ABSTRACT}

Q2 This cross-sectional study examined binge eating and binge drinking behaviors exploring their association with the strategies of Emotion Regulation, such as Cognitive Reappraisal (CR) and Expression Suppression (ES). The sample consisted of 1004 Italian students (395 males and 609 females) attending high school. The average age of the students was 17.9 years (SD $=0.8$; range: $16-21$ ). They completed self-report measures assessing binge behaviors and Emotion Regulation strategies. No differences resulted between adolescents assuming binge behaviors and no bingers as regards CR; conversely, adolescents engaging in binge eating and in both binge behaviors reported the highest levels of ES. Furthermore, both Emotion Regulation strategies were significant predictors of binge eating. The present study suggested that binge behaviors were strongly associated with adolescent's ability to evaluate and manage their emotions, so that adolescents may benefit from more precise and specific prevention and treatment approaches focused on training to use more adaptive and effective strategies to regulate their emotions.

\section{KEYWORDS}

Binge eating; binge

drinking; emotion

regulation; adolescence
55

56

57

58

59

60

61

62

63

64

65

66

67

68

69

70

Binge drinking and binge eating can be considered real "addictive" behaviors as uncontrolled and repetitive consumption of either alcohol or food effectively associated with physical, social and psychological difficulties. ${ }^{1-4}$ As established in previous research, binge drinking is defined as the consumption of five or more consecutive drinks for men and four or more consecutive drinks for women on a single occasion. ${ }^{5-8}$ Binge eating is defined as the consumption of a larger amount of food than usual during a limited period of time (e.g.) within an hour) accompanied by experiencing a feeling of marked distress and loss of control over eating. ${ }^{9}$ Binge drinking and binge eating represent the two most frequently observed behaviors within the range of mood-altering appetitive behaviors that indicate risk to both current and future health and wellbeing during adolescence. Particularly in the Italian context, occasional alcohol consumption among adolescents has been increasing (from $38.8 \%$ in 2006 to $43.4 \%$ in 2016) and $35 \%$ of adolescents reported to binge drink in the past month (37\% of males and $33 \%$ of females). ${ }^{10}$ The main risk factor for binge drinking episodes among adolescents is the involvement in social activities such as concerts, sporting events and clubs. ${ }^{11}$ Indeed, the high prevalence of binge drinking, reflects a significant change in alcohol use among young people during the last decades which is characterized by a transition from moderate daily alcohol consumption during meals (typical of Mediterranean model) to a growing episodic and excessive alcohol consumption outside of meals time (typical of Northern European countries). ${ }^{12,13}$ Furthermore, estimates suggest that binge eating is quite widespread among young people aged 15 to 19 years, and $70 \%$ of them are females. ${ }^{14}$

Indeed, adolescence is a period of life characterized by a great expression of risk-taking and the tendency to experience new behaviors as a normative part of developmental processes; however, most adolescents tend to underestimate negative consequences directly associated with binge behaviors. Binge eating and binge drinking

CONTACT Fiorenzo Laghi fiorenzo.laghi@uniroma1.it Department of Developmental and Social Psychology, University of Rome Sapienza, Rome 00185, Italy. 
are also frequently shared among individuals in the same peer network reflecting the high tendency of adolescents make social comparisons and to adopt a certain course of action sanctioned by their peer group and not personal preferences. ${ }^{15,16}$ In considering why adolescents repeatedly engage in binge behaviors, it has been argued that they serve a similar function and purpose such as regulating emotions and/or escaping from negative emotions and coping with distress. ${ }^{17-21}$ Above all, women who binge eat and drink often report the two behaviors in response to feeling lonely, bored or powerless in relation to their partners or they describe bingeing as a means of escape from fear of failure or feelings of low self-esteem and inadequacy. ${ }^{22-24}$ Indeed, recent studies showed that binge eating and binge drinking among young people were associated with difficulties in emotion regulation (ER) and in dealing with situations involving negative emotions. ${ }^{25-27}$ Specifically, Whiteside et $\mathrm{al}^{27}$ found that binge eating in college students was related to difficulties in identifying and making sense of emotions and with a limited access to ER strategies. Thus, alcohol or food are substances that can be used by young people to regulate emotions, and drinking or eating may be perceived as a strategy of ER in order to influence emotional affect. ${ }^{28,29}$ Although there is a growing interest in analyzing emotional factors underlying the engagement in binge behaviors, only few studies have examined the role of ER in binge eating and binge drinking among adolescents. For instance, the important role of coping (or emotional) motives has been highlighted as the main triggering factor of binge eating among adolescents ${ }^{20}$; particularly, it has been noted that binge eaters tended to use such behavior to avoid or reduce perceived negative affective states. On the other hand, binge drinking was predicted by enhancement motives, such that adolescents, rather than managing aversive states, engaged in binge drinking to have fun, to seek excitement, to make a party more enjoyable, and to get high and drunk. Thus, binge behaviors, and especially binge eating, may function as a way to forget worries and provide comfort and distraction from negative affect. However, few studies currently exist on this topic among adolescents and, to our knowledge, no study has explored together

both ER strategies for binge eating and binge drinking.

In line with these considerations, the analysis of regulation strategies in adolescence is crucial to better understand individual differences in adolescents' engagement in binge behaviors, possibly providing indicators for the prevention of these dysfunctional behaviors. ER can be defined as the processes by which individuals alter and control emotional experience modifying and influencing when and how specific emotions are expressed and how emotions are experienced ${ }^{13}$; more specifically, Gross ${ }^{30}$ suggested a process-oriented model including specifying two different ER strategies: 1) cognitive reappraisal (CR), that is, an antecedent focused strategy including the attempt to think about the situation and the possibility to change or reformulate its meaning and the emotional impact; 2) expressive suppression (ES), that is, a response focused strategy constituted by efforts to restrain or inhibit external facial, bodily or behavioral sign of emotions. These two strategies are differently related to psychological functioning and well-being. ${ }^{30-33}$ Indeed, CR relates to greater experience of positive affect, better relationship closeness and less adverse consequences for either memory or emotional responsiveness in social interactions, while ES tends to be associated with less experience and expressions of positive emotions and social support and greater levels of negative affect and depressive symptoms. ${ }^{34-36}$ In addition, a great and chronic use of ES is a risk factor for a variety of dysfunctional behaviors including alcohol use and disordered eating. ${ }^{37,38}$ Several studies have found the ineffectiveness of ES in altering emotional experience and reducing negative effect such as fear and disgust; ${ }^{39,40}$ thus, ES has been described as a supposed maladaptive strategy in regulating emotions. ${ }^{37,38}$ Conversely, CR has shown to be an effective strategy in altering affect in experimental studies and has been negatively associated with health-risk behaviors, although difficulties in reappraisal have shown a weaker association with dysfunctional behaviors than $\mathrm{ES}^{37}$; therefore, $\mathrm{CR}$ appears to be a more adaptive ER strategy for adolescents' well-being. 
The present study aimed to analyze the relation between ER and binge behaviors during adolescence. Specifically, in light of previous findings, we hypothesized that ER strategies-CR and ES-would discriminate no binge adolescents, binge eaters, binge drinkers, and binge eaters and drinkers. In particular, in line with previous studies, ${ }^{37,38}$ which highlighted that a high level of ES was associated with dysfunctional eating and drinking behaviors, we hypothesized that binge eaters and drinkers would tend to report higher levels of ES.

\section{Methods}

\section{Sample}

The sample consisted of 1004 students (395 males and 609 females). The average age of the students was 17.9 ( $\mathrm{SD}=0.8$; range $16-21$ ). Participants involved in the study were in the last two years of high school and the majority of them were aged 17-18 years (80.8\%). We conducted this study in 14 schools, seven from the Center and seven from South of Italy. Three additional schools declined to participate in the study. Schools' selection was based on their willingness to take part in the current research. Each school was contacted by sending letters where objectives and procedures of the study were explained. Although data regarding the cultural origin of the participants were not available, we estimated $97 \%$ were of majority Italian culture. High schools in Italy are divided into distinct educational and occupational fields and include five grade levels. In terms of type of high school, $39.3 \%$ were in technical-industrial arts schools $(n=395), 22.6 \%$ were in teacher-training schools $(n=227), 21.7 \%$ of students were enrolled in science-focused schools $(n=218), 9.8 \%$ were in liberal artsfocused schools $(n=98)$ and $6.6 \%$ were in classic high schools $(n=66)$.

\section{Procedures}

Participation was preceded by an informed-consent procedure that required active consent from both students and parents. The questionnaires were administered in the classroom during a normal class and took approximately $45 \mathrm{~min}$. to complete. Instructions stated that the questionnaires were voluntary and that responses were anonymous and confidential. All participants returned and responded to the administered questionnaire; of them, $96 \%$ fully completed all the required measures. This survey was reviewed and approved by the Ethics Commission of the Department of Developmental and Social Psychology of Sapienza, University of Rome.

\section{Measures}

Binge eating scale

To measure the severity of binge eating, the binge eating scale (BES) was administered. ${ }^{41}$ The BES is a 16-item self-report questionnaire designed to capture both behavioral manifestations of binge eating (eight items, e.g., eating large amounts of food) and cognitive and emotional characteristics associated with a binge episode (eight items, e.g., guilt and fear of being unable to stop eating). Each item comprises three or four weighted statement reflecting a range of severity for each characteristic, and respondents are asked to select the one which best describes them. Weights are from 0 to 3 where 0 reflects no binge eating problems and 3 indicates severe binge eating problems; for instance, item number two has four statements that are: 1) I don't have any difficulty eating slowly in the proper manner (weight $=0)$; 2) Although I seem to "gobble down" foods, I don't end up feeling stuffed because of eating too much (weight $=1$ ). 3) At times, I tend to eat quickly and then, I feel uncomfortably full afterwards (weight $=2$ ); 4) I have the habit of bolting down my food, without really chewing it. When this happens I usually feel uncomfortably stuffed because I've eaten too much (weight $=3$ ). The BES is scored by adding the individual values for the 16 items resulting in a possible range of scores from 0 to 46 , with higher scores indicating more severe binge eating symptoms. ${ }^{41,42}$ While the BES is typically used in obese or clinical binge eating disorder populations, the questionnaire has also been used in normal-weight samples $^{43}$ to categorize participants into binge eating and non-binge eating groups. Previous studies $^{44-47}$ have used a cutoff score of 17 on the BES to classify individuals with binge eating 
Table 1. Differences in drinking characteristics, binge eating, and emotion regulation according to sex.

\begin{tabular}{lcrrc}
\hline & Females $(N=609) M(S D)$ & Males $(N=395) M(S D)$ & $F(1,1002)$ & Partial Eta \\
\hline BMI self-reported & $21.81(3.32)$ & $22.97(3.36)$ & $26.52^{*}$ & 0.03 \\
Frequency of alcohol consumption (0-4) & $2.16(0.85)$ & $2.75(0.97)$ & $103.23^{*}$ & 0.09 \\
Drinks per occasion & $3.40(1.64)$ & $4.58(2.07)$ & $100.88^{*}$ & 0.09 \\
Age at onset of alcohol use & $14.66(2.62)$ & $13.65(2.59)$ & $33.72^{*}$ & 0.03 \\
Age when they first became intoxicated & $15.97(1.83)$ & $15.38(1.79)$ & $14.43^{*}$ & 0.03 \\
Times gotten drunk in the last month & $0.21(0.62)$ & $0.55(1.45)$ & $24.53^{*}$ & 0.02 \\
Getting drunk after drinking (\% times) & $10.90(21.23)$ & $17.90(25.34)$ & $21.40^{*}$ & 0.02 \\
Binge drinking (number of episodes) & $0.35(0.80)$ & $0.70(1.41)$ & $23.76^{*}$ & 0.02 \\
Binge eating scale (Total score) & $9.98(6.79)$ & $7.06(6.38)$ & $46.52^{*}$ & 0.04 \\
Cognitive reappraisal (ERQ) & $20.91(4.98)$ & $20.47(4.47)$ & 2.43 & 0.00 \\
Expressive suppression (ERQ) & $11.55(3.48)$ & $11.40(3.34)$ & 0.43 & 0.00 \\
\hline
\end{tabular}

symptoms; thus, based on the BES total score, individuals scoring 17 or less are characterized by no binge eating symptoms and those with a score of 18 or above are classified as binge eaters. In accordance with other studies in the Italian context, ${ }^{43,48-50}$ we used the BES total score as a continuous measure of binge eating tendencies, and (separately) a score of 18 and above as categorial measure of binge eating in order to classify adolescents with no-binge eating symptoms and binge eaters. ${ }^{46,51}$

The BES translation has been validated by the NetWorking Team Group of the Italian Society for Eating Behavior Disorders. ${ }^{43}$ The BES has good test-retest reliability $(r=0.87, p<.001)$ and moderate associations with binge eating severity as measured by food records $(r=0.20-0.40, p<$ $.05)^{42}$. Internal consistency for the BES in the current investigation was satisfactory $(\alpha=0.83)$.

\section{Alcohol use/severity}

Drinking quantity and frequency were assessed using several questions: a) frequency of alcohol consumption $(0=$ Never; $1=$ Less than once a month/once a month; $2=$ Two/four times a month; $3=$ Two/Three times a week; $4=$ Four or more times a week); b) average number of drinks consumed per occasion; c) age when regular drinking began; d) age when first intoxication episode occurred; e) number of times gotten drunk in the last month and $\mathrm{f}$ ) percentage of times getting drunk when drinking. The specific wording of the question concerning binge drinking was as follows: "Considering all types of alcoholic beverage, did you have five or more drinks (four if you are female) on one single occasion

Note: ${ }^{*} p<.001$. Partial eta-squared values were calculated as a measure of effect size, and results were interpreted using Cohen's (1988) guidelines for determining small (0.01), medium (0.06), and large (0.14) effects.

during the past two weeks? Alcohol use/severity variables were used in the analyses carried out in our study for both descriptive purposes and as covariates in regression analyses as potential confounders of the relation between ER strategies and binging behaviors.

\section{ER questionnaire}

The ERQ ${ }^{32}$ comprises 10 items assessing the ER strategies of CR (six items) and ES (ES; four items). The Italian version of ERQ has been reported to have high internal consistency, as well as sound convergent and discriminant validity with both younger and older adults. ${ }^{34}$ The ERQ was also found to be a reliable and useful tool in assessing ER in adolescents. ${ }^{52}$ According to Gullone and Taffe, ${ }^{35}$ we reduced the response scale length to five points $(1=$ strongly disagree, $2=$ disagree,$\quad 3=$ half and half, $4=$ agree, $5=$ strongly agree). The range of scores for each scale was 6 to 30 for the CR and 4 to 20 for the ES. A reliability analysis on the two subscales indicated good levels of internal consistency (Cronbach's $\alpha=0.70$ for CR; Cronbach's $\alpha=0.81$ for ES).

\section{Results}

Preliminary analyses: Sex and geographical area differences

A series of ANOVA tests revealed only sex differences and not geographical differences in alcohol use, binge eating behavior, and ER dimensions. Males obtained higher scores than females for BMI, frequency of alcohol consumption, drinks per occasion, number of times the subject became 
Table 2. Correlations among the variables.

\begin{tabular}{|c|c|c|c|c|c|c|c|c|c|c|c|c|}
\hline Variable & 1. & 2. & 3. & 4. & 5. & 6. & 7. & 8. & 9. & 10. & 11. & 12. 13 \\
\hline 1. Sex $(0=$ females $1=$ males $)$ & - & & & & & & & & & & & \\
\hline 2. Age & 0.04 & - & & & & & & & & & & \\
\hline 3. BMI self-reported & $0.17 * *$ & 0.01 & - & & & & & & & & & \\
\hline 4. Frequency of alcohol consumption (0-4) & $0.31^{* *}$ & 0.06 & $0.13^{*}$ & $=$ & & & & & & & & \\
\hline 5. Drinks per occasion & $0.30 * *$ & 0.05 & $0.13^{*}$ & $0.89 * *$ & - & & & & & & & \\
\hline 6. Age at onset of alcohol use & $-0.19 * *$ & 0.07 & -0.07 & $-0.16 * *$ & $-0.16^{* *}$ & $=$ & & & & & & \\
\hline 7. Age when they first became intoxicated & $-0.16^{* *}$ & 0.09 & $-0.11 *$ & $-0.16^{* *}$ & $-0.16^{* *}$ & $0.41^{* *}$ & ${ }^{k}-$ & & & & & \\
\hline 8. Times gotten drunk in the last month & $0.16^{* *}$ & 0.06 & 0.04 & $0.35^{* *}$ & $0.35^{* *}$ & $-0.13^{* *}$ & $-0.18^{* *}$ & & & & & \\
\hline 9. Getting drunk after drinking (\% times) & $0.15^{* *}$ & 0.02 & -0.02 & $0.39 * *$ & $0.38^{* *}$ & $-0.13^{* *}$ & ${ }^{k}-0.18^{* *}$ & $0.44 * *$ & & & & \\
\hline 10. Binge drinking (number of episodes) & $0.15^{* *}$ & 0.03 & 0.09 & $0.42 * *$ & $0.42^{* *}$ & $-0.10^{*}$ & $-0.14 * *$ & $0.53^{* *}$ & $0.33^{* *}$ & - & & \\
\hline 11. Binge eating scale (Total score) & $-0.21^{* *}$ & 0.04 & $0.16^{*}$ & 0.06 & 0.06 & 0.04 & 0.03 & 0.06 & $0.13^{* *} \mathrm{c}$ & 04 & - & \\
\hline 12. Cognitive reappraisal (ERQ) & -0.05 & 0.00 & -0.09 & -0.04 & -0.03 & -0.01 & -0.05 & 0.00 & 0.01 & $-0.02-$ & -0.05 & - \\
\hline 13. Expressive suppression (ERQ) & -0.02 & -0.01 & 0.00 & -0.03 & -0.03 & -0.03 & -0.05 & 0.08 & 0.07 & 0.03 & $0.16^{* *}$ & $0.12^{* *}-$ \\
\hline
\end{tabular}

drunk in the last month, number of binge drinking episodes in the last two weeks, and getting drunk after drinking. Males were younger than females when they began to drink on regular basis, and when they got intoxicated for the first time. Females obtained higher scores than males for binge eating, as shown in Table 1.

\section{Correlations among study variables}

Pearson correlations were performed to examine the relationships among the key variables used in the present study: variables related to alcohol use, binge eating behavior, and ER dimensions. Only binge eating was related to $\mathrm{ER}$, as reported in Table 2.

\section{Binge group membership}

On the basis of their responses to the binge screening measure, adolescents were assigned to one of four binge behavior groups: binge drinking only (males who consumed 5 or more consecutive drinks and females who consumed 4 or more consecutive drinks on a single occasion within the past two weeks; $N=227 ; 22.6 \%$ ), binge eating only (adolescents who obtained a score of 18 and above on BES; $N=89 ; 8.9 \%$ ), both behaviors (binge eating and drinking; $N=37 ; 3.7 \%$ ), and neither binge behavior (no binge; $N=651 ; 64.8 \%)$. In a series of chi-square tests, we examined whether there were significant differences among the four binge behavior groups with respect to geographical area, sex, and type of school. Significant differences were found for sex $[\chi 2=(3)=23.45, p<.001]$, and for type of school $\left[\chi^{2}=(12)=29.12, p=.004\right]$. Females were more likely to be classified as binge eaters $(11 \%$ of females vs. $5.6 \%$ of males), while males were more likely to be classified as binge drinkers ( $29.6 \%$ of males vs. $18.1 \%$ of females), such that males reported more binge drinking than females, and the latter showed more binge eating than males. Students who attended technical-industrial arts schools were more likely to be classified as binge drinkers (31.3\%), while students who attended teacher training schools were more likely to be classified as binge eaters $(29.2 \%)$, and as binge drinker and eaters (32.4\%).

\section{$E R$ and binge group classification}

The Analysis of Covariance of binge group classifications as a between-subjects factor, and sex as covariate, was conducted for the ERQ dimensions. Sex, used as a covariate, did not show significant covariations with groups for $\mathrm{CR}(\mathrm{F}(1,1002)=2.82$; $p=.09)$, and for ES $(\mathrm{F}(1,1002)=0.22 ; p=.63)$. The analysis revealed a significant effect for ES $\left(\mathrm{F}(3,1000)=3.01 ; p=.02 ; \eta_{p} 2=0.009\right)$, and not for $\mathrm{CR}(\mathrm{F}(3,1000)=0.90 ; p=.44)$. Results from the univariate tests and post-hoc test (Tukey test; $p$ $<.05)$ revealed that binge eat $(M=12.30$; SD $=3.22$ ) and binge eat and drink group $(M=12.46 ; \mathrm{SD}=3.03)$ had significantly higher mean levels of ES than the binge drink group $(M=11.45 ; \mathrm{SD}=3.41)$ and no binge group $(M=11.34 ; \mathrm{SD}=3.46)$ that did not differ significantly from either.

\section{The role of ER in predicting binge drinking and binge eating}

In order to examine the role of ER as predictor of binge behaviors (measured continuously), hierarchical regression analyses were conducted. In
452

453

454

455

456

457

458

459

460

461

462

463

464

465

466

467

468

469

470

471

472

473

474

475

476

477

478

479

480

481

482

483

484

485

486

487

488 
Table 3. Hierarchical regression analysis for variables predicting binge drinking.

\begin{tabular}{lccccccc}
\hline Predictor & $B$ & $S E B$ & $\beta$ & $R^{2}$ & $\Delta R^{2}$ & Df & $\Delta F$ \\
\hline$\quad$ Step 1 & & & & 0.02 & 0.02 & 1,499 & $10.26^{* *}$ \\
Sex $(0=$ females 1 = males) & 0.39 & 0.12 & $0.14^{* *}$ & & & & \\
$\quad$ Step 2 & & & & 0.09 & 0.07 & 3,496 & $13.13^{* *}$ \\
$\quad$ Age when they first became intoxicated & -0.08 & 0.04 & $-0.10^{*}$ & & & & \\
$\quad$ Age at onset of alcohol use & -0.002 & 0.03 & -0.003 & & & & \\
$\quad$ Getting drunk after drinking (\% times) & 0.01 & 0.002 & $0.23^{* *}$ & & & & \\
Step 3 & & & & 0.09 & 0.00 & 2,494 & 0.10 \\
$\quad$ Cognitive reappraisal (ERQ) & -0.005 & 0.01 & -0.08 & & & & \\
$\quad$ Expressive suppression (ERQ) & 0.004 & 0.02 & 0.01 & & & & \\
\hline Note: $* p<.05, * * p \leq .001$. & & & & & & &
\end{tabular}

steps 1 and 2, we have included demographic variables and alcohol use variables, which correlated with binge drinking or binge eating as covariates. In step 3, ER strategies were entered. For binge drinking as the dependent variable, we entered sex (females $=0$, males $=1$ ) in step 1 . Findings indicated that sex significantly predicted binge drinking, $\beta=0.14, p=.001$. In the following step, alcohol use variables were added to the model, the age when they first became intoxicated, the age at onset of alcohol use, and percentage of times getting drunk when drinking. Sex remained a significant predictor once these variables were added, $\beta=0.11, p<.05$. Alcohol use significantly predicted binge drinking, $R^{2}=$ $0.09, F(4,496)=12.60, p=.001$, with age when they first became intoxicated emerging as a significant predictor of binge drinking, $\beta=-0.10, p$ $<.05$, as well as percentage of times getting drunk when drinking, $\beta=0.23, p<.001$. In step 3 , ER strategies were added to the model; neither $\mathrm{CR}$ nor ES were related to binge drinking (Table 3).

For binge eating as the dependent variable, regression analysis was performed in three steps, sex (coded as 1 for males and 0 for females) and BMI were entered in step 1 as covariates. Consistent with previous studies, ${ }^{53,54}$ we wanted to control for any confounding influence of BMI on the relationship between binge eating and ER strategies. Our results revealed that both sex and BMI were significant predictors of binge eating accounting for $9 \%$ of the variance. Percentage of times getting drunk when drinking was entered in step 2 emerged as a significant predictor, $\beta=0.18, p<.001$. CR, $\beta=-.09, p<.01$ and ES, $\beta=0.16, p<.001$ added to the model at step
3 , were both significant predictors of binge eating (Table 4).

\section{Discussion}

Adolescents experience more frequent and intense emotion than younger individuals, and their capacity to manage or regulate emotions in a socially appropriate and adaptive manner has been recognized as a central risk factor for both binge eating and drinking. ${ }^{17,18}$ Our findings tended to confirm that emotional strategies that are response focused, as Expression Suppression in the model suggested by Gross, ${ }^{30}$ seem to characterize adolescents who engage in binge behaviors. Consistent with this theory, higher levels of ES were reported by binge eaters and adolescents assuming both binge behaviors, but not by binge drinkers. This result seems to support the idea that youths who binge eat may use this behavior as a way to regulate and manage strong emotional states; indeed, it has been noted that binge behaviors are related to high levels of negative affect and expectancies that drinking or eating behaviors may help to improve mood. ${ }^{1}$ Thus, experiencing intense negative emotions and the inability to express them may increase susceptibility to binge eating and also to assume both binge behaviors in vulnerable adolescents. In other studies ES has been linked to increased caloric consumption following negative mood induction in obese individuals. ${ }^{55}$ Furthermore, previous research also demonstrated that situations involving negative emotional states posed the highest risk for engaging in binge eating or in both binge behaviors, while situations involving interpersonal interactions or pleasant times 
Table 4. Hierarchical regression analysis for variables predicting binge eating.

\begin{tabular}{lrrrrrrr}
\hline Predictor & $B$ & $S E B$ & $\beta$ & $R^{2}$ & $\Delta R^{2}$ & $\mathrm{~d} f$ & $\Delta F$ \\
\hline Step 1 & & & & 0.09 & 0.09 & 2,895 & $44.05^{* *}$ \\
$\quad$ Sex $(0=$ females 1=males) & -3.59 & 0.45 & $-0.26^{* *}$ & & & & \\
$\quad$ BMI & 0.39 & 0.06 & $0.20^{* *}$ & & & & \\
Step 2 & & & & 0.12 & 0.03 & 1,894 & $33.22^{* *}$ \\
$\quad$ Getting drunk after drinking (\% times) & 0.05 & 0.01 & $0.18^{* *}$ & 0.15 & 0.03 & 2,892 & $15.31^{* *}$ \\
Step 3 & & & & 0.09 & 0.07 & 3,496 & $13.13^{* *}$ \\
$\quad$ Cognitive reappraisal (ERQ) & -0.14 & 0.05 & $-0.09^{*}$ & & & & \\
$\quad$ Expressive suppression (ERQ) & 0.31 & 0.06 & $0.16^{* *}$ & & & & \\
\hline Note: ${ }^{*} p<.01, * * p<.001$. & & & & & &
\end{tabular}

with others lead adolescents to assume binge drinking behaviors. More specifically, Birch et al. ${ }^{17}$ argue that binge eating amongst women is a relief from psychological distress (e.g., reducing anxiety and desires) and temptation, while binge drinking is more likely to occur in reward situations (e.g., fulfilling needs and desires).

Thus, it could be plausible that binge drinking may not necessarily represent a way to escape from negative emotion or to manage them, but simply a way to have time with peers. Indeed, many adolescents declared to drink for fun, to be happy, to gain confidence, to be cool or simply for something to do. Furthermore, positive alcohol expectancies, or the belief that drinking will lead to positive experiences (e.g., increasing in sociability or reducing social anxiety) seem to be significant predictors of binging among young people. ${ }^{6,56}$ This could explain the reason why binge drinkers did not significantly differ in levels of Expression Repression from non-bingers.

Furthermore, both ES and CR were significant predictors of binge eating, further highlighting difficulties for binge eaters to express their emotional states. In line with previous studies, ${ }^{57,58}$ our results suggest that adolescents who binge eat are characterized by an increased use of maladaptive ER strategies such as ES and a reduced use of a more adaptive strategy as CR to manage their emotions. Indeed, it has been noted ${ }^{37}$ that, both the lack of adaptive strategies and the presence of maladaptive strategies may increase the risk for eating disorder symptoms. ${ }^{37,38}$ Thus, the experience of frequent or strong negative affect is not problematic in itself; individuals who experience more intense emotions and know how to regulate them may not show unfavorable consequences. It may be that the lack of ER skills can induce binge eaters to learn to use such dysfunctional behavior as a coping strategy. ${ }^{29,59-61}$ Indeed, earlier experimental studies $55,57,62$ showed how training in CR to regulate negative emotions reduced binge eating behaviors; conversely, using ES to manage negative emotional states, resulted in more food consumption. Thus, our data support the argument that binge eaters may have difficulties in managing emotions especially when they are faced with negative ones and are characterized by a great use of maladaptive ER strategies, as they have not learnt more adaptive and functional ways to regulate them.

The lack of association between binge drinking and ER strategies may be attributed to a more complex relationship, which may involve the role of other variables. For instance, reward sensitivity may moderate this relation, as individuals with both high level of reward sensitivity and difficulties in regulating emotions may be at increased risk to use alcohol as a way to alter and regulate affect; ${ }^{37}$ indeed, an association has been found among young adults between higher reward sensitivity and earlier age of onset of alcohol use. ${ }^{37,63}$

There are some limitations to our study. First, the cross-sectional design tested in this study restricted our ability to establish the direction of the associations we examined. Second, this study was performed on a school-based sample of Italian adolescents; this may limit the generalizability of our findings. Future research should consider the implementation of a longitudinal design, which could help to better understand the temporal nature of the study variables and to test the course and potential long-term effects of the 
process of ER, as well as the employment of ecological momentary assessment. Moreover, future studies are also needed in order to better clarify the relationship between ER and binge behaviors; particularly, it could be useful to consider other variables that could be involved in this relationship, such as negative affect, as existing literature suggests that binge eating and binge drinking may serve the same purpose, that is to reduce or avoid negative emotional states. Indeed, it may be interesting to examine how ER strategies interact with negative affect in order to better clarity whether it can reinforce binging behaviors and contribute to their onset and maintenance. In addition, future directions may involve the investigation of the role of reward sensitivity as a moderator in the relation between ER strategies and binge drinking.

Despite these limitations, the present study provides an important contribution to the literature regarding binging behaviors; unlike the majority of research on the topic, which has mainly involved college students, our study included a sample of adolescents in high school. Furthermore, to our knowledge, this was the first study focused on investigating ER strategies both for binge drinking and binge eating. Our findings highlighted how adolescents who engage in bingeing and, especially in binge eating, may have difficulties in regulating their emotions and may use substances (alcohol or food) as a coping strategy in order to enhance their unregulated affect. Specifically, the present study suggests how a great use of maladaptive ER strategies may be an important risk factor for binging behaviors and thus, it highlights the importance of training adolescents in using more adaptive strategies, in order to properly respond to intense emotional situations. In this respect, findings from this research may be useful from an applied perspective, as improving ER can be one way to intervene with and prevent binging behaviors. ${ }^{64}$ Therefore, future programs may be oriented to help adolescents learn to accept emotions nonjudgementally and develop and practice functional ER strategies in order to manage intense and negative emotions. Specifically, adolescents who engage in binge behaviors, may benefit from
Dialectical Behavior Therapy (DBT) that has demonstrated its efficacy in individuals with problems regulating emotions and impulsivity such as binges. ${ }^{65-67}$ Indeed, DBT may train adolescents in accepting emotional states that may be perceived as unpleasant. Mindfulness is a core part of DBT that may help them to tolerate and accept intense and negative emotions, such as sadness, anger, loneliness and fully experience their emotions without judgment.

\section{ORCID}

Fiorenzo Laghi (D) http://orcid.org/0000-0003-3969-4292

Francesca Liga (D) http://orcid.org/0000-0001-7074-6711

\section{References}

1. Ferriter C, Ray LA. Binge eating and binge drinking: An integrative review. Eat Behav 2011;12:99-107.

2. Laghi F., Liga F, Baumgartner E, Baiocco R. Identity and conformism among Italian adolescents who binge eat and drink. Health Risk Soc 2012;14(4):361-76. doi:10.1080/13698575.2012.680952.

3. Laghi F, Baiocco R, Liga F, Lonigro A, Baumgartner E. Binge eating and binge drinking bahaviors: Individual differences in adolescents' identity styles. J Health Psychol 2014;19(3):333-43. doi:10.1177/ 1359105312470851.

4. Martin JL, Groth G, Longo L, Rocha TL, Martens MP. Disordered eating and alcohol use among college women: associations with race and big five traits. Eat Behav 2015;17:149-52. doi:10.1016/j.eatbeh.2015.02.002.

5. Agius P, Taft A, Hemphill S, Toumbourou J, McMorris B. Excessive alcohol use and its association with risky sexual behaviour: a cross-sectional analysis of data from Victorian secondary school students. Aust N Z J Public Health 2013;37(1):76-82. doi: 10.1111/1753-6405.12014.

6. McBride NM, Barrett B, Moore KA, Schonfeld L. The role of positive alcohol expectancies in underage binge drinking among college students. J Am Coll Health 2014;62(6): 370-9. doi:10.1080/07448481.2014.907297.

7. Wechsler $H$, Nelson TF. Binge drinking and the American college student: what's five drinks? Psychol Addict Behav 2001;15(4): 287-91.

8. Wechsler H, Davenport A, Dowdall G, Moeykens B, Castillo S. Health and behavioral consequences of binge drinking in college: A national survey of students at 140 campuses. Jama 1994;272(21): 1672-7. doi:10.1001/jama.1994.03520210056032.

9. American Psychiatric Association. Diagnostic and StatisticalManual of Mental Disorders, 5th edition 
(DSM-5). Arlington, VA: American Psychiatric Association, 2013: 350-3.

10. European School Survey Projection on Alcohol and Other Drugs, 2015. Available: http://www.espad.org/ sites/espad.org/files/ESPAD_report_2015.pdf

11. ISTAT. Alcohol Consumption in Italy, 2016. Available: https://www.istat.it/en/files/2017

12. Allamani A, Beccaria F, Voller F. The puzzle of Italian drinking. Nord Stud Alcohol Dr 2010;27(5): 465-78. doi:10.1177/145507251002700504.

13. Beccaria F, Prina F. Young people and alcohol in Italy: An evolving relationship. Drugs 2010;17(2): 99-122. doi:10.3109/09687630802291703.

14. Favaro A, Ferrara S, Santonastaso P. The spectrum of eating disorders in young women: A prevalence study in a general population sample. Psychosom Med 2003; 65:701-8. doi:10.1097/01.PSY.0000073871.67679.D8.

15. Balsa AL, Homer JF, French MT, Norton EC. Alcohol use and popularity: Social payoffs from conforming to peers' behavior. J Res Adolesc 2010;21(3):559-68.

16. Laghi F, Baumgartner E, Baiocco R, Kotzalidis GD, Piacentino D, Girardi P, Angeletti G. Alcohol intake and binge drinking among Italian adolescents: The role of drinking motives. J Addict Dis 2016;35(2): 119-27. doi:10.1080/10550887.2015.1129703.

17. Birch CD, Sherry HS, Brown CG. Exploring differential patterns of situational risk for binge eating and heavy drinking. Addict Behav 2017;32:433-48. doi: 10.1016/j.addbeh.2006.05.014.

18. Kenny TE, Singleton C, Carter JC. Testing prediction of the emotion regulation model of binge-eating disorder. Int J Eat Disord 2017;50(11):1297-305. doi: 10.1002/eat.22787.

19. Laghi F, Liga F, Baumgartner E, Baiocco R. Time perspective and psychosocial positive functioning among Italian adolescents who binge eat and drink. J Adolesc 2012;35:1277-84. doi:10.1016/j.adolescence.2012.04.014.

20. Pompili S, Laghi F. Binge eating and binge drinking among adolescents: The role of drinking and eating motives. J Health Psychol. Epub 2017 June 8. doi: https://doi.org/10.1177/135910531771335.

21. Pompili S, Laghi F, Drunkorexia among adolescents: The role of motivations and emotion regulation. Eat Behav. Epub 2018 Jan 16. doi: https://doi.org/10.1016/ j.eatbeh.2018.01.001.

22. Corstorphine E, Waller G, Ohanian V, Baker M. Changes in internal states across the binge-vomit cycle in bulimia nervosa. J Nerv Ment Dis 2006;194(6): 446-449. doi:10.1097/01.nmd.0000221303.64098.23.

23. Davis R, Jamieson J. Assessing the functional nature of binge eating in the eating disorders. Eat Behav 2005;6(4): 345-54.

24. Heatherton TF, Baumeister RF. Binge eating as escape from self-awareness. Psychol Bull 1991;110(1); 86-108.

25. Czaja J, Rief W, Hilbert A. Emotion regulation and binge eating in children. Int J Eat Disord 2009;42(4): 356-62.
26. Lavender JM, Anderson DA. Contribution of emotion regulation difficulties to disordered eating and body dissatisfaction in college men. Int J Eat Disord 2010; 43(4): 352-7.

27. Whiteside U, Chen E, Neighbors C, Hunter D, Lo T, Larimer M. Difficulties regulating emotions: Do binge eaters have fewer strategies to modulate and tolerate negative affect? Eat Behav 2007;8(2): 162-9.

28. Boggiano MM, Burgess EE, Turan B, Soleymani T, Daniel S, Vinson, LD, et al. Motives for eating tasty foods associated with binge-eating. Results from a student and a weight-loss seeking population. Appetite 2014: 83: 160-6. doi:10.1016/j.appet.2014.08.026.

29. Veilleux JC, Skinner KD, Reese ED, Shaver JA. Negative affect intensity influences drinking to cope through facets of emotion dysregulation. Pers Individ Dif 2014;59: 96-101. doi:10.1016/j.paid.2013.11.012.

30. Gross JJ. The emerging field of emotion regulation: An integrative review. Rev Gen Psychol 1998;2:271-99. doi: 10.1037/1089-2680.2.3.271.

31. Gross JJ. Emotion regulation in adulthood: Timing is everything. Curr Dir Psychol Sci 2001;10:214-9. doi: 10.1111/1467-8721.00152.

32. Gross JJ, John OP. Individual differences in two emotion regulation processes: Implications for affect, relationships, and wellbeing. J Pers Soc Psychol 2003; 85:348-62. doi:10.1037/0022-3514.85.2.348.

33. Srivastava S, Tamir M, McGonigal KM, John OP, Gross JJ. The social costs of emotional suppression: A prospective study of the transition to college. J Pers Soc Psychol 2009;96:883-97

34. Balzarotti S, John OP, Gross JJ. An Italian adaptation of the Emotion Regulation Questionnaire. Eur J Psychol Assess 2010;26(1):61-7. doi:10.1027/1015-5759/a000009.

35. Gullone E, Taffe J. The emotion regulation questionnaire for children and adolescents (ERQ-CA): A psychometric evaluation. Psychol Assess 2012;24(2): 409-17. doi:10.1037/a0025777.

36. Verzeletti C, Zammuner VL, Galli C, Agnoli S. Emotion regulation strategies and psychosocial wellbeing in adolescence. Cogent Psychol 2015;3:1-15.

37. Aldao A, Nolen-Hoeksema S, Schweizer S. Emotionregulation strategies across psychopathology: A metaanalytic review. Clin Psychol Rev 2010;30(2): 217-37. doi:10.1016/j.cpr.2009.11.004.

38. Aldao A, Jazaieri H, Goldin PR, Gross JJ Adaptive and maladaptive emotion regulation strategies: Interactive effects during CBT for social anxiety disorder. J Anxiety Disord 2014;28(4): 382-9. doi:10.1016/ j.janxdis.2014.03.005.

39. Gross JJ. Emotion regulation: Affective, cognitive, and social consequences. Psychophysiology 2002;39(3): 281-91.

40. Sheppes G, Gross JJ. Emotion regulation effectiveness: What works when. Handbook of psychology 2012;2: 391-406. 
41. Gormally J, Black S, Daston S, Rardin D. The assessment of binge eating severity among obese subjects. Addict Behav 1982;7:47-55. doi:10.1016/0306-4603(82)90024-7.

42. Timmerman GM. Binge eating scale: Further assessment of validity and reliability. J App Biobehav Res 1999;4(1):1-12. doi:10.1111/j.1751-9861.1999.tb00051.x.

43. Dalle Grave R, Calugi S, Coriga F, Di Domizio S, Marchesini G, QUOVADIS Study Group. Psychological variables associated with weight loss in obese patients seeking treatment at Medical Centers. J Am Diet Assoc 2009;109: 2010-6. doi:10.1016/j.jada.2009.09.011.

44. Marcus MD, Wing RR, Hopkins J. Obese binge eaters: affect, cognitions, and response to behavioral weight control. J Consult Clin Psychol 1988;56(3): 433-9. doi:10.1037/0022-006X.56.3.433.

45. Ricca V, Mannucci E, Moretti S, Di Bernardo M, Zucchi $\mathrm{T}$, Cabras PL, et al. Screening for binge eating disorder in obese outpatients. Compr Psychiatry 2000;41: 111-5.

46. Greeno CG, Marcus MD, Wing RR. Diagnosis of binge eating disorder: discrepancies between a questionnaire and clinical interview. Int J Eat Disord 1995; 2:153-60. doi:10.1002/1098-108X(199503)17:2<153:: AID-EAT2260170208>3.0.CO;2-V.

47. Grupski AE, Hood MM, Hall BJ, Azarbad L, Fitzpatrick SL, Corsica JA. Examining the binge eating scale in screening for binge eating disorder in bariatric surgery candidates. Obes Surg 2013;23(1): 1-6.

48. Dalle Grave R, Calugi S, Petroni ML, Di Domizio S, Marchesini G, QUOVADIS Study Group. Weight management, psychological distress and binge eating in obesity. A reappraisal of the problem. Appetite 2010;54:269-73. doi:10.1016/j.appet.2009.11.010.

49. Mannucci E, Petroni ML, Villanova N, Rotella CM, Apolone G, Marchesini G, et al. Clinical and psychological correlates of health-related quality of life in obese patients. Health Qual Life Outcomes 2010 (online). Available: www.hqlo.com/content/8/1/90. doi:10. 1186/1477-7525-8-90.

50. Imperatori $\mathrm{C}$, Innamorati $\mathrm{M}$, Lamis DA, Contardi A, Continisio M, Castelnuovo G, et al. Factor structure of the binge eating scale in a large sample of obese and overweight patients attending low energy diet therapy. Eur Eat Disord Rev 2016;24(2): 174-8. doi: 10.1002/erv.2384.

51. Freitas SR, Lopes CS, Appolinario JC, Coutinho W. The assessment of binge eating disorder in obese women: A comparison of the binge eating scale with the structured clinical interview for the DSM-IV. Eat Behav 2006;7: 282-9 doi:10.1016/j.eatbeh.2005.09.002.

52. Laghi F, Lonigro A, Pallini S, Baiocco R. Emotion regulation and empathy: Which relation with social conduct? J Genet Psychol 2018;179(2): 62-70.

53. Stice E, Presnell K, Spangler D. Risk factors for binge eating onset in adolescent girls: A 2-year prospective investigation. Health Psychol 2002;21(2): 131-8. doi: 10.1037/0278-6133.21.2.131.
54. Fitzgibbon ML, Spring B, Avellone ME, Blackman, LR, Pingitore R, Stolley MR. Correlates of binge eating in Hispanic, Black, and White women. Int J Eat Disord 1998;24(1): 43-52.

55. Svaldi J, Griepenstroh J, Tushen-Caffier B, Ehring T. Emotion regulation deficits in eating disorders: a marker of eating pathology or general psychopathology? Psychiatry Res 2012;197:103-11.

56. Biolcati R, Passini S, Mancini G. "I cannot stand the boredom." Binge drinking expectancies in adolescence. Addict Behav Rep 2016;3:70-6. doi:10.1016/ j.abrep.2016.05.001.

57. Svaldi J, Caffier D, Tuschen-Caffier B. Emotion suppression but not reappraisal increases desire to binge in women with binge eating disorder. Psychother Psychosom 2010;79(3): 188-190.

58. Dingemans A, Danner U, Parks M. Emotion regulation in binge eating disorder: A review. Nutrients 2017;9(11): 1274. doi:10.3390/nu9111274.

59. Pompili S, Laghi F. Drunkorexia: Disordered eating behaviors and risky alcohol consumption among adolescents. J Health Psychol. 2018. doi: https://doi.org/ $10.1177 / 1359105318791229$

60. Laghi F, Bianchi D, Pompili S, Lonigro A, Baiocco R. Metacognition, emotional functioning and binge eating in adolescence: the moderation role of need to control thoughts. Eat Weight Disord Epub 2018 Oct 27. doi: https://doi.org/10.1007/s40519-018-0603-1

61. Laghi F, Bianchi D, Pompili S, Lonigro A, Baiocco R. Cognitive and affective empathy in binge drinking adolescents: Does empathy moderate the effect of selfefficacy in resisting peer pressure at drinking? Addict Behav. Epub 2018 Oct 11. doi: https://doi.org/10. 1016/j.addbeh.2018.10.015

62. Vohs KD, Heatherton TF. Self-regulatory failure: A resource-depletion approach. Psychol Sci 2010;11(3): 249-54.

63. Pardo Y, Aguilar R, Molinuevo B, Torrubia R. Alcohol use as a behavioural sign of disinhibition: Evidence from J.A. Gray's model of personality. Addict Behav 2007;32: 2398-2403.

64. Han S, Lee S. College student binge eating: Attachment, psychological needs satisfaction, and emotion regulation. J Coll Stud Dev 2017;58(7): 1074-86. doi:10.1353/csd.2017.0084.

65. Telch CF, Agras WS, Linehan MM. Dialectical behavior therapy for binge eating disorder. J Consult Clin Psychol 2001;69(6): 1061-5.

66. Wiser S, Telch CF . Dialectical behavior therapy for binge-eating disorder. J Clin Psychol 1999;55(6): 755-68.

67. Zgierska A, Rabago D, Chawla N, Kushner K, Koehler $\mathrm{R}$, Marlatt A. Mindfulness meditation for substance use disorders: a systematic review. Subst Abus 2009; 30(4): 266-94. 